\title{
Supporting Information \\ Morphological Impact of Segment Dispersity in \\ Lithium Salt-Doped Poly(styrene)/Poly(ethylene \\ oxide) Triblock Polymers
}

Hongyun Xu,† Eric M. Greve§ $\uparrow$ and Mahesh K. Mahanthappa†**

$\dagger$ Department of Chemical Engineering \& Materials Science, University of Minnesota, 421

Washington Ave. S.E., Minneapolis, MN 55455

$\S$ Department of Chemistry, University of Wisconsin-Madison, 1101 University Ave., Madison, WI 53706

I Present address: Department of Chemistry, Marquette University, Milwaukee, WI 53233

* Corresponding author: maheshkm@umn.edu; +1 (612) 625-4599.

\begin{tabular}{|l|c|}
\hline Table of Contents & Page \\
\hline $\begin{array}{l}\text { Figure S1. Temperature dependent 1D-SAXS patterns of bSOS_38.0_0.38 showing } \\
\text { disordering at elevated temperature. }\end{array}$ & S2 \\
\hline Table S1. Lamellar $d$-spacings of salt-doped bSOS and nSOS at $r=0.01$. & $\mathrm{S} 3$ \\
\hline Table S2. Lamellar $d$-spacings of salt-doped bSOS and nSOS at $r=0.05$. & $\mathrm{S} 4$ \\
\hline Table S3. Lamellar $d$-spacings of salt-doped bSOS and nSOS at $r=0.09$. & $\mathrm{S} 5$ \\
\hline $\begin{array}{l}\text { Figure S2. Lamellar } d \text {-spacing comparison between bSOS and nSOS triblock } \\
\text { polymers at increased salt loading. }\end{array}$ & $\mathrm{S} 6$ \\
\hline $\begin{array}{l}\text { Figure S3. Lamellar } d \text {-spacing and the power-law fitting of narrow dispersity SO } \\
\text { diblock polymers at } r=0.085 .\end{array}$ & $\mathrm{S} 7$ \\
\hline References & $\mathrm{S} 8$ \\
\hline
\end{tabular}




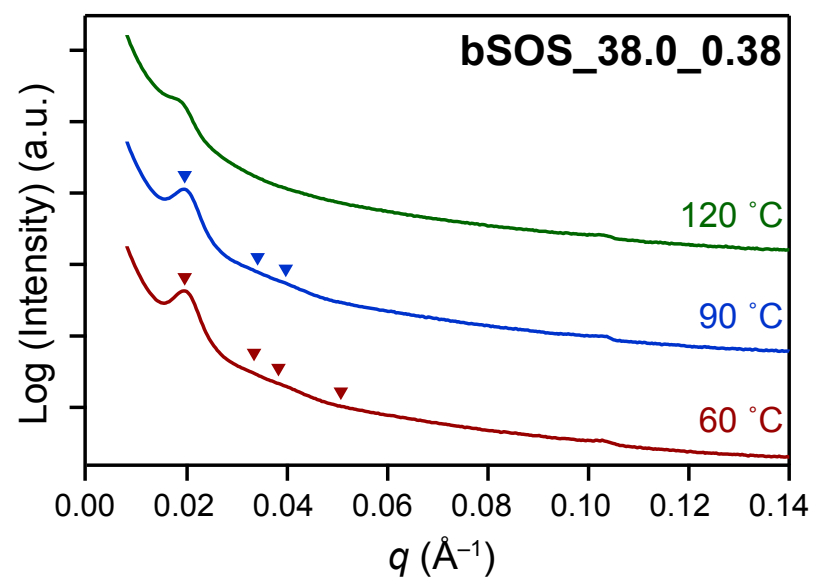

Figure S1. One-dimensional $I(q)$ versus scattering wavevector $q$ synchrotron SAXS profiles the microphase separated yet poorly ordered triblock polymer bSOS_38.0_0.38, for which higher order reflections disappear upon heating to $120{ }^{\circ} \mathrm{C}$. Note that the peak at $q=0.104 \AA^{-1}$ is an artifact associated with the detector mask. 
Table S1. Lamellar $d$-spacings of Salt-Doped bSOS and nSOS Triblocks at $r=0.01$.

\begin{tabular}{ccc} 
Sample & $N^{\mathrm{a}}$ & $d^{\mathrm{b}}(\mathrm{nm})$ \\
\hline bSOS_19.6_0.63 & 308 & 19.7 \\
bSOS_21.2_0.70 & 332 & 20.5 \\
bSOS_24.8_0.60 & 390 & 20.3 \\
\hline nSOS_19.0_0.53 & 300 & 14.1 \\
nSOS_20.0_0.50 & 316 & 14.7 \\
nSOS_42.6_0.47 & 673 & 23.1 \\
nSOS_45.2_0.44 & 715 & 24.5
\end{tabular}

${ }^{\mathrm{a}}$ Degree of polymerization calculated at $90{ }^{\circ} \mathrm{C}$. ${ }^{\mathrm{b}}$ Calculated from the primary scattering peak position $\left(q^{*}\right)$ at $90{ }^{\circ} \mathrm{C}$ using $d=2 \pi / q^{*}$. 
Table S2. Lamellar $d$-spacings of Salt-Doped bSOS and nSOS Triblocks at $r=0.05$.

\begin{tabular}{ccc} 
Sample & $N^{\mathrm{a}}$ & $d^{\mathrm{b}}(\mathrm{nm})$ \\
\hline bSOS_11.6_0.63 & 182 & 13.8 \\
bSOS_12.2_0.60 & 192 & 12.4 \\
bSOS_12.8_0.58 & 202 & 14.2 \\
bSOS_18.4_0.70 & 289 & 18.8 \\
bSOS_19.6_0.66 & 308 & 20.3 \\
bSOS_21.2_0.72 & 332 & 21.0 \\
bSOS_23.2_0.56 & 366 & 21.1 \\
bSOS_23.9_0.55 & 377 & 20.2 \\
bSOS_24.8_0.63 & 390 & 21.0 \\
\hline nSOS_11.2_0.44 & 177 & 10.0 \\
nSOS_19.0_0.56 & 300 & 16.6 \\
nSOS_20.0_0.53 & 316 & 16.9 \\
nSOS_23.2_0.46 & 348 & 18.7 \\
nSOS_22.0_0.48 & 367 & 18.3 \\
nSOS_42.6_0.50 & 673 & 26.9 \\
nSOS_45.2_0.47 & 715 & 27.8 \\
\hline
\end{tabular}

${ }^{\text {a }}$ Degree of polymerization calculated at $90{ }^{\circ} \mathrm{C}$. ${ }^{\mathrm{b}}$ Calculated from the primary scattering peak position $\left(q^{*}\right)$ at $90{ }^{\circ} \mathrm{C}$ using $d=2 \pi / q^{*}$. 
Table S3. Lamellar $d$-spacings of Salt-Doped bSOS and nSOS Triblocks with $r=0.09$.

\begin{tabular}{|c|c|c|}
\hline Sample & $N^{\mathrm{a}}$ & $d^{\mathrm{b}}(\mathrm{nm})$ \\
\hline bSOS_11.7_0.55 & 185 & 13.6 \\
\hline bSOS_11.6_0.66 & 182 & 14.4 \\
\hline bSOS_12.2_0.63 & 192 & 12.5 \\
\hline bSOS_12.8_0.61 & 202 & 14.9 \\
\hline bSOS_18.4_0.72 & 289 & 18.7 \\
\hline bSOS_19.6_0.69 & 308 & 21.5 \\
\hline bSOS_20.9_0.65 & 329 & 21.1 \\
\hline bSOS_21.2_0.75 & 332 & 21.8 \\
\hline bSOS_23.2_0.59 & 366 & 22.3 \\
\hline bSOS_23.9_0.58 & 377 & 21.8 \\
\hline bSOS_24.8_0.65 & 390 & 21.9 \\
\hline nSOS_7.1_0.52 & 112 & 8.3 \\
\hline nSOS_8.4_0.60 & 132 & 9.1 \\
\hline nSOS_11.2_0.47 & 177 & 10.6 \\
\hline nSOS_11.8_0.45 & 187 & 10.9 \\
\hline nSOS_12.8_0.41 & 203 & 11.9 \\
\hline nSOS_15.0_0.36 & 239 & 12.7 \\
\hline nSOS_19.0_0.58 & 300 & 16.8 \\
\hline nSOS_20.0_0.56 & 316 & 16.8 \\
\hline nSOS_22.0_0.51 & 348 & 19.6 \\
\hline nSOS_23.2_0.49 & 367 & 19.4 \\
\hline nSOS_42.6_0.53 & 673 & 29.3 \\
\hline nSOS_45.2_0.50 & 715 & 30.5 \\
\hline
\end{tabular}

${ }^{\mathrm{a}}$ Degree of polymerization calculated at $90{ }^{\circ} \mathrm{C} .{ }^{b}$ Calculated from the primary scattering peak position ( $\left.q^{*}\right)$ at $90{ }^{\circ} \mathrm{C}$ using $d=2 \pi / q^{*}$. 


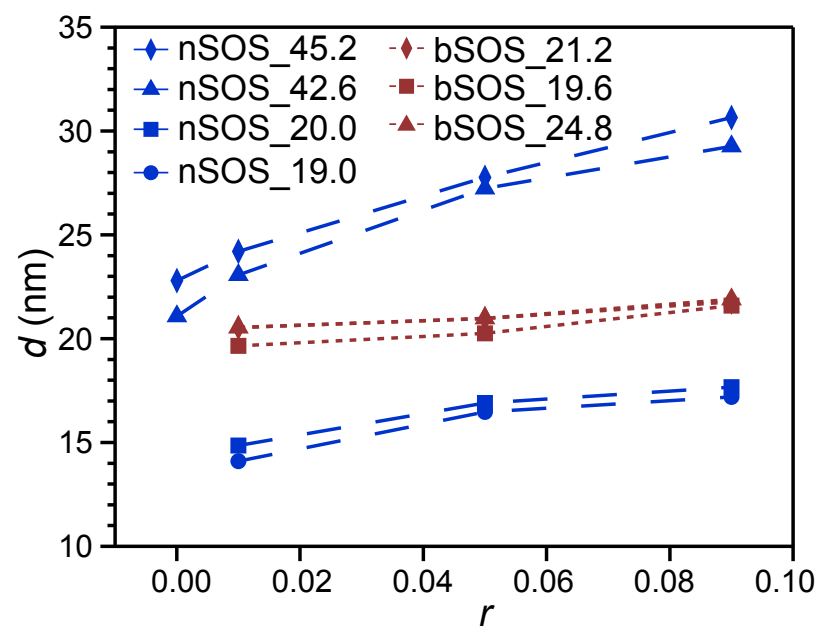

Figure S2. Lamellar $d$-spacing of salt-doped bSOS and nSOS with salt loadings $0 \leq r \leq 0.09$. 


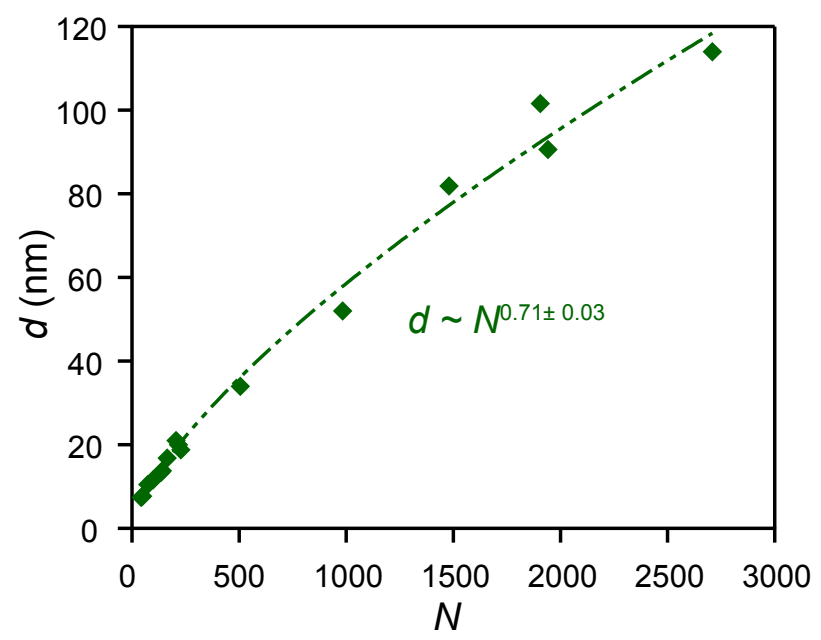

Figure S3. Lamellar $d$-spacing of narrow dispersity SO diblock polymers at $r=0.085$ from Panday et al. ${ }^{1}$ and Yuan et al. ${ }^{2}$ The dashed line represents the fit to $d \sim N^{0.71 \pm 0.03}$. 


\section{Reference}

1. Panday, A.; Mullin, S.; Gomez, E. D.; Wanakule, N.; Chen, V. L.; Hexemer, A.; Pople, J.; Balsara, N. P., Effect of Molecular Weight and Salt Concentration on Conductivity of Block Copolymer Electrolytes. Macromolecules 2009, 42, 4632-4637.

2. Yuan, R.; Teran, A. A.; Gurevitch, I.; Mullin, S. A.; Wanakule, N. S.; Balsara, N. P., Ionic Conductivity of Low Molecular Weight Block Copolymer Electrolytes. Macromolecules 2013, 46, 914-921. 